\title{
CZĘSTOŚĆ DRGAŃ WŁASNYCH SŁUPA O ZMIENNYM PRZEKROJU POPRZECZNYM*
}

\author{
Vazgen Bagdasaryan ${ }^{凶}$ \\ Wydział Budownictwa i Inżynierii Środowiska, Szkoła Główna Gospodarstwa Wiejskiego w Warszawie, Warszawa
}

\begin{abstract}
STRESZCZENIE
W pracy wyznaczone zostały częstości drgań własnych wspornikowego słupa o zmiennym przekroju poprzecznym, wykonanego z jednorodnego materiału sprężystego. Rozwiązania otrzymano metodą macierzy sztywności, przybliżając w sposób inżynierski ciągłą zmianę przekroju poprzecznego na zmiany skokowe. Otrzymane częstości drgań własnych porównano z wynikami otrzymanymi metodą Rayleigh’a. Wykazano, iż stosunkowo proste obliczenia inżynierskie już przy niewielkiej liczbie elementów podziału dają wyniki satysfakcjonujące pod względem zastosowań inżynierskich.
\end{abstract}

Słowa kluczowe: częstość drgań własnych, zmienny przekrój poprzeczny, macierz sztywności

\section{WSTĘP}

Częstość drgań własnych odgrywa kluczową rolę podczas projektowania konstrukcji inżynierskich szczególnie w przypadku słupów, masztów, kominów itp. Ze względów inżynierskich wpływ drgań na konstrukcje powinien być uwzględniany przy sprawdzaniu stanu granicznego użytkowalności. W literaturze jest wiele metod pozwalających wyznaczyć częstości drgań własnych konstrukcji. Bardzo ciekawe są zawsze wyniki ścisłe, do których można odnosić otrzymane rozwiązania przybliżone, a wśród nich np. metody oparte na metodzie funkcji Greena (Szewczyk, Kukla i Zamojska, 2007) czy też ścisłe rozwiązania równania Eulera-Bernoulliego (Naguleswaran, 1994). Istnieją również liczne metody przybliżone, a wśród nich metody numeryczne, którymi zajmował się m.in. Caruntu (2009), wyznaczając częstości drgań własnych belek o zmiennym przekroju poprzecznym. Metodą Rayleigh'a wyznaczania częstości drgań własnych słupów o zmiennym przekroju poprzecznym zajmowali się np. Bagdasaryan, Chalecki, Gierasimiuk, Jaworski i Szlachetka (2018). Celem niniejszej pracy jest wykazanie, iż rozwiązania otrzymane inżynierską metodą zamiany obliczeń słupa o ciagłej zmianie przekroju poprzecznego na obliczenia słupa o skokowo zmiennym przekroju dają wyniki satysfakcjonujące pod względem zastosowań inżynierskich już przy niewielkiej liczbie elementów podziału.

\section{MATERIA $~ I$ METODY}

W pracy rozważono jednostronnie utwierdzony słup o zmiennym przekroju poprzecznym w kształcie ściętego stożka (rys. 1). Założono dla niego gęstość masy $\left(\rho_{s}\right)$ oraz moduł odkształcenia podłużnego $\left(E_{s}\right)$.

\footnotetext{
* Due to complexity of the article text was formatted in one-column page style.

Vazgen Bagdasaryan https://orcid.org/0000-0002-9589-1453

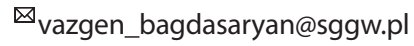




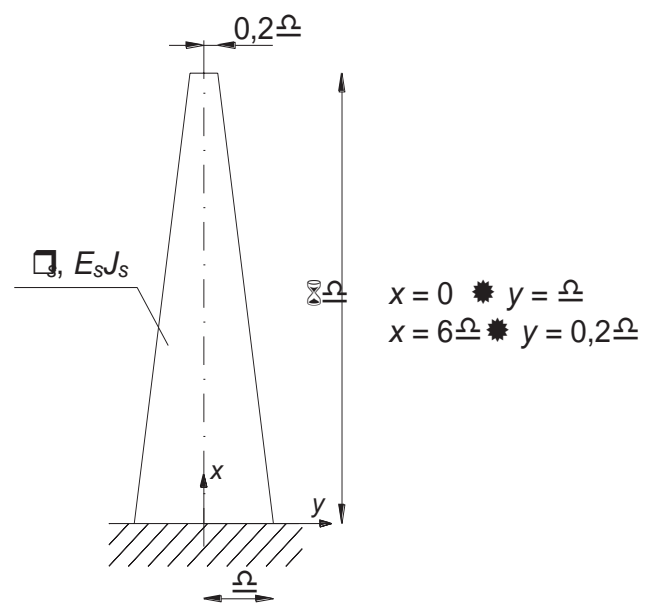

Rys. 1. Schemat rozpatrywanego słupa o zmiennym przekroju poprzecznym

Fig. 1. Diagram of the considered pole with variable cross-section

W dalszej części rozpatrzono pięć przypadków podziału rozpatrywanego słupa na jedna, dwie, trzy, cztery oraz pięć części o równych długościach i o stałym, kołowym przekroju poprzecznym (rys. 2), przyjmując promienie wyznaczonych części jako wartość średnią z długości podziału. Masy prętów zostały uwzględniane, dlatego też stosuje się tzw. metodę ,przez sztywność" nazwaną w pracy metodą macierzy sztywności. Macierze sztywności dla wybranych słupów otrzymano, stosując metodę przemieszczeń.

\section{Wariant 1 - słup o stałym przekroju poprzecznym}

Dla słupa z rysunku 2a wyznaczono niezbędne do obliczeń wielkości:

$$
J_{1}^{1}=\frac{\pi\left(\delta_{1}^{1}\right)^{4}}{4}=\frac{\pi}{4}\left(\frac{3}{5} \delta\right)^{4}=0,0324 \pi \delta^{4} ;
$$

$\mu_{1}^{1}=\rho_{s} \cdot A_{1}^{1}=\rho_{s} \cdot \pi \cdot\left(\delta_{1}^{1}\right)^{2}=\rho_{s} \cdot \pi \cdot\left(\frac{3}{5} \delta\right)^{2}=0,36 \pi \rho_{s} \delta^{2}$. a)

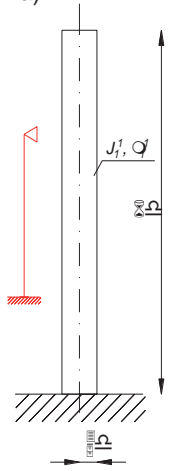

b)

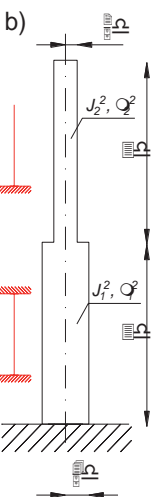

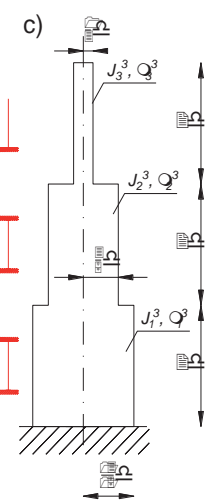

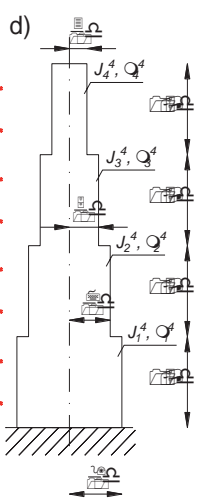

Rys. 2. Schemat wariantów uproszczonych o stałym przekroju poprzecznym: a - podział na jedną część; b - podział na dwie części; c - podział na trzy części; d - podział na cztery części; e - podział na pięć części

Fig. 2. Diagram of simplified variants with constant cross-section: $a$ - division into one part; $b$ - division into two parts; $\mathrm{c}$ - division into three parts; $\mathrm{d}$ - division into four parts; $\mathrm{e}$ - division into five parts 
Założono dalej $E_{s} J_{1}^{1}=E J, \mu_{1}^{1}=\mu$.
Dla całego słupa wyznaczono parametr $v_{1}^{1}=6 v$, gdzie $v=\delta \sqrt[4]{\frac{\mu \omega^{2}}{E J}}$.

Ze względu na konieczność wprowadzenia niewiadomej metody przemieszczeń rozpatrywany słup przedstawiono jako schemat utwierdzenie-podparcie (niewiadomy przesuw swobodnego końca słupa).

Macierz sztywności ma w tym wypadku prostą postać

$$
\mathbf{M}=\left[0,0046 \chi^{\prime}(6 v)\right], \text { gdzie } \chi^{\prime}(v)=v^{3} \frac{1+\cosh v \cos v}{\cosh v \sin v-\sinh v \cos v} .
$$

Warunkiem wyznaczenia częstości drgań własnych jest zerowanie się wyznacznika macierzy sztywności, co prowadzi tutaj do wyznaczenia równania $0,0046 \chi^{\prime}(6 v)=0$.

\section{Wariant 2 - podział słupa na dwie części}

Dla słupa z rysunku $2 \mathrm{~b}$ wyznaczono niezbędne do obliczeń wielkości:

$$
\begin{aligned}
& J_{1}^{2}=\frac{\pi\left(\delta_{1}^{2}\right)^{4}}{4}=\frac{\pi}{4}\left(\frac{4}{5} \delta\right)^{4}=0,1024 \pi \delta^{4} \\
& J_{2}^{2}=\frac{\pi\left(\delta_{2}^{2}\right)^{4}}{4}=\frac{\pi}{4}\left(\frac{2}{5} \delta\right)^{4}=0,0064 \pi \delta^{4}
\end{aligned}
$$

Przy założeniu $E_{s} J_{1}^{2}=E J$ mamy $E_{s} J_{2}^{2}=0,063 E J$.

$$
\begin{aligned}
& \mu_{1}^{2}=\rho_{s} \cdot A_{1}^{2}=\rho_{s} \cdot \pi \cdot\left(\delta_{1}^{2}\right)^{2}=\rho_{s} \cdot \pi \cdot\left(\frac{4}{5} \delta\right)^{2}=0,64 \pi \rho_{s} \delta^{2} ; \\
& \mu_{2}^{2}=\rho_{s} \cdot A_{2}^{2}=\rho_{s} \cdot \pi \cdot\left(\delta_{2}^{2}\right)^{2}=\rho_{s} \cdot \pi \cdot\left(\frac{2}{5} \delta\right)^{2}=0,16 \pi \rho_{s} \delta^{2} ;
\end{aligned}
$$

Analogicznie przy założeniu $\mu_{1}^{2}=\mu$ mamy $\mu_{2}^{2}=0,25 \mu$.

Dla poszczególnych części słupa wyznaczono parametry $v_{1}^{2}=3 v$ oraz $v_{2}^{2}=4,2 v$.

W przypadku podziału słupa na dwie części o stałych przekrojach macierz sztywności ma postać

$$
\mathbf{M}=\left[\begin{array}{cc}
0,333 \alpha(3 v)+0,021 \alpha^{\prime \prime}(4,2 v) & -0,111 \vartheta(3 v)+0,007 \vartheta "(4,2 v) \\
-0,111 \vartheta(3 v)+0,007 \vartheta "(4,2 v) & 0,037 \gamma(3 v)+0,002 \gamma^{\prime \prime}(4,2 v)
\end{array}\right],
$$

gdzie:

$$
\begin{array}{ll}
\alpha(v)=v \frac{\cosh v \sin v-\sinh v \cos v}{1-\cosh v \cos v} ; & \vartheta^{\prime \prime}(v)=v^{2} \frac{-\sinh v \sin v}{1+\cosh v \cos v} ; \\
\alpha^{\prime \prime}(v)=v \frac{\sinh v \cos v-\cosh v \sin v}{1+\cosh v \cos v} ; & \gamma(v)=v^{3} \frac{\cosh v \sin v+\sinh v \cos v}{1-\cosh v \cos v} ; \\
\vartheta(v)=v^{2} \frac{\sinh v \sin v}{1-\cosh v \cos v} ; & \gamma^{\prime \prime}(v)=-v^{3} \frac{\sinh v \cos v+\cosh v \sin v}{1+\cosh v \cos v} ;
\end{array}
$$




\section{Wariant 3 - podział słupa na trzy części}

Dla słupa z rysunku 2c wyznaczono niezbędne do obliczeń wielkości

$$
\begin{aligned}
& J_{1}^{3}=\frac{\pi\left(\delta_{1}^{3}\right)^{4}}{4}=\frac{\pi}{4}\left(\frac{13}{15} \delta\right)^{4}=0,1410 \pi \delta^{4} ; \\
& J_{2}^{3}=\frac{\pi\left(\delta_{2}^{3}\right)^{4}}{4}=\frac{\pi}{4}\left(\frac{3}{5} \delta\right)^{4}=0,0324 \pi \delta^{4} \\
& J_{3}^{3}=\frac{\pi\left(\delta_{3}^{3}\right)^{4}}{4}=\frac{\pi}{4}\left(\frac{1}{3} \delta\right)^{4}=0,0031 \pi \delta^{4}
\end{aligned}
$$

Przy założeniu $E_{s} J_{1}^{3}=E J$ mamy $E_{s} J_{2}^{3}=0,230 E J$ oraz $E_{s} J_{3}^{3}=0,021 E J$.

$$
\begin{aligned}
& \mu_{1}^{3}=\rho_{s} \cdot A_{1}^{3}=\rho_{s} \cdot \pi \cdot\left(\delta_{1}^{3}\right)^{2}=\rho_{s} \cdot \pi \cdot\left(\frac{13}{15} \delta\right)^{2}=0,751 \pi \rho_{s} \delta^{2} \\
& \mu_{2}^{3}=\rho_{s} \cdot A_{2}^{3}=\rho_{s} \cdot \pi \cdot\left(\delta_{2}^{3}\right)^{2}=\rho_{s} \cdot \pi \cdot\left(\frac{3}{5} \delta\right)^{2}=0,360 \pi \rho_{s} \delta^{2} \\
& \mu_{3}^{3}=\rho_{s} \cdot A_{3}^{3}=\rho_{s} \cdot \pi \cdot\left(\delta_{3}^{3}\right)^{2}=\rho_{s} \cdot \pi \cdot\left(\frac{1}{3} \delta\right)^{2}=0,111 \pi \rho_{s} \delta^{2} .
\end{aligned}
$$

Analogicznie przy założeniu $\mu_{1}^{3}=\mu$ otrzymujemy $\mu_{2}^{3}=0,48 \mu$ oraz $\mu_{3}^{3}=0,15 \mu$.

Dla poszczególnych części słupa wyznaczono parametry: $v_{1}^{3}=2 v, v_{2}^{3}=2,4 v$ oraz $v_{3}^{3}=3,3 v$.

W przypadku podziału słupa na trzy części o stałych przekrojach macierz sztywności ma postać

$$
\mathbf{M}=\left[\begin{array}{cccc}
0,5 \alpha(2 v)+0,115 \alpha(2,4 v) & 0,115 \beta(2,4 v) & -0,058 \delta(2,4 v) & -0,25 \vartheta(2 v)+0,058 \vartheta(2,4 v) \\
0,115 \beta(2,4 v) & 0,115 \alpha(2,4 v)+0,012 \alpha^{\prime \prime}(3,3 v) & 0,005 \vartheta "(3,3 v)-0,058 \vartheta(2,4 v) & 0,058 \delta(2,4 v) \\
-0,058 \delta(2,4 v) & 0,005 \vartheta "(3,3 v)-0,058 \vartheta(2,4 v) & 0,029 \gamma(2,4 v)+0,003 \gamma "(3,3 v) & -0,029 \varepsilon(2,4 v) \\
-0,25 \vartheta(2 v)+0,058 \vartheta(2,4 v) & 0,058 \delta(2,4 v) & -0,029 \varepsilon(2,4 v) & 0,125 \gamma(2 v)+0,029 \gamma(2,4 v)
\end{array}\right],
$$

gdzie:

$$
\begin{aligned}
& \beta(v)=v \frac{\sinh v-\sin v}{1-\cosh v \cos v} \\
& \delta(v)=v^{2} \frac{\cosh v-\cos v}{1-\cosh v \cos v} \\
& \varepsilon(v)=v^{3} \frac{\sin v+\sinh v}{1-\cosh v \cos v}
\end{aligned}
$$




\section{Wariant 4 - podział słupa na cztery części}

Dla słupa z rysunku 2d wyznaczono niezbędne do obliczeń wielkości:

$$
\begin{aligned}
& J_{1}^{4}=\frac{\pi\left(\delta_{1}^{4}\right)^{4}}{4}=\frac{\pi}{4}\left(\frac{9}{10} \delta\right)^{4}=0,1640 \pi \delta^{4} ; \\
& J_{2}^{4}=\frac{\pi\left(\delta_{2}^{4}\right)^{4}}{4}=\frac{\pi}{4}\left(\frac{7}{10} \delta\right)^{4}=0,0600 \pi \delta^{4} ; \\
& J_{3}^{4}=\frac{\pi\left(\delta_{3}^{4}\right)^{4}}{4}=\frac{\pi}{4}\left(\frac{5}{10} \delta\right)^{4}=0,0156 \pi \delta^{4} ; \\
& J_{4}^{4}=\frac{\pi\left(\delta_{4}^{4}\right)^{4}}{4}=\frac{\pi}{4}\left(\frac{3}{10} \delta\right)^{4}=0,0020 \pi \delta^{4} .
\end{aligned}
$$

Przy założeniu $E_{s} J_{1}^{4}=E J$ mamy: $E_{s} J_{2}^{4}=0,366 E J, E_{s} J_{3}^{4}=0,095 E J$ oraz $E_{s} J_{4}^{4}=0,012 E J$.

$$
\begin{aligned}
& \mu_{1}^{4}=\rho_{s} \cdot A_{1}^{4}=\rho_{s} \cdot \pi \cdot\left(\delta_{1}^{4}\right)^{2}=\rho_{s} \cdot \pi \cdot\left(\frac{9}{10} \delta\right)^{2}=0,81 \pi \rho_{s} \delta^{2} \\
& \mu_{2}^{4}=\rho_{s} \cdot A_{2}^{4}=\rho_{s} \cdot \pi \cdot\left(\delta_{2}^{4}\right)^{2}=\rho_{s} \cdot \pi \cdot\left(\frac{7}{10} \delta\right)^{2}=0,49 \pi \rho_{s} \delta^{2} \\
& \mu_{3}^{4}=\rho_{s} \cdot A_{3}^{4}=\rho_{s} \cdot \pi \cdot\left(\delta_{3}^{4}\right)^{2}=\rho_{s} \cdot \pi \cdot\left(\frac{5}{10} \delta\right)^{2}=0,25 \pi \rho_{s} \delta^{2} \\
& \mu_{4}^{4}=\rho_{s} \cdot A_{4}^{4}=\rho_{s} \cdot \pi \cdot\left(\delta_{4}^{4}\right)^{2}=\rho_{s} \cdot \pi \cdot\left(\frac{3}{10} \delta\right)^{2}=0,09 \pi \rho_{s} \delta^{2} .
\end{aligned}
$$

Analogicznie przy założeniu $\mu_{1}^{4}=\mu$ otrzymujemy: $\mu_{2}^{4}=0,605 \mu, \mu_{3}^{4}=0,309 \mu$ oraz $\mu_{4}^{4}=0,111 \mu$.

Dla poszczególnych części słupa wyznaczono parametry: $v_{1}^{4}=1,5 v, v_{2}^{4}=1,7 v, v_{3}^{4}=2,0 v$ oraz $v_{4}^{4}=2,6 v$.

W przypadku podziału słupa na cztery części o stałych przekrojach macierz sztywności ma postać

gdzie:

$$
\mathbf{M}=\left[m_{i j}\right] \text {, dla } i, j=1,2,3,4,5,6,
$$

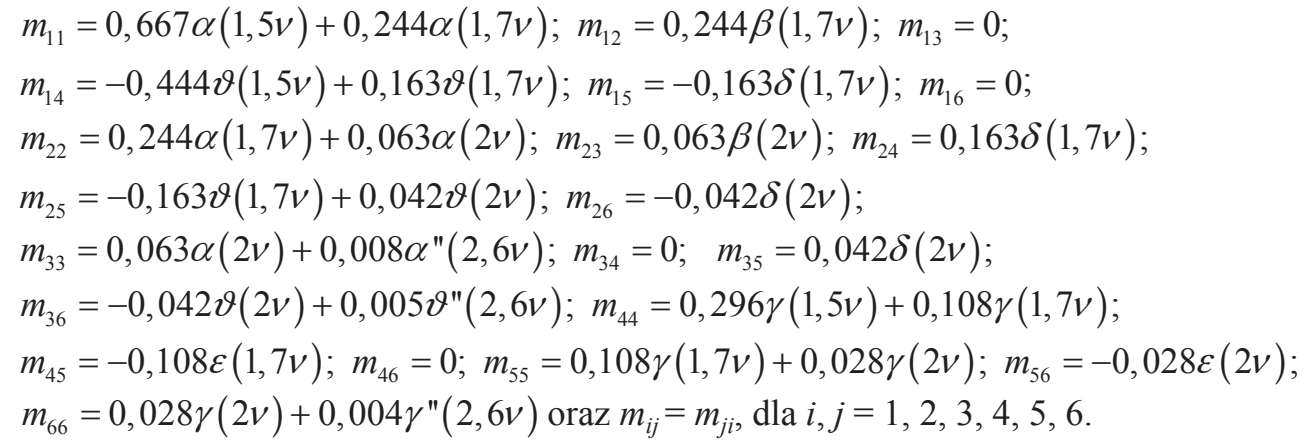




\section{Wariant 5 - podział słupa na pięć części}

Dla słupa z rysunku 2e wyznaczono niezbędne do obliczeń wielkości:

$$
\begin{aligned}
& J_{1}^{5}=\frac{\pi\left(\delta_{1}^{5}\right)^{4}}{4}=\frac{\pi}{4}(0,92 \delta)^{4}=0,1797 \pi \delta^{4} ; \\
& J_{2}^{5}=\frac{\pi\left(\delta_{2}^{5}\right)^{4}}{4}=\frac{\pi}{4}(0,76 \delta)^{4}=0,0834 \pi \delta^{4} ; \\
& J_{3}^{5}=\frac{\pi\left(\delta_{3}^{5}\right)^{4}}{4}=\frac{\pi}{4}(0,6 \delta)^{4}=0,0324 \pi \delta^{4} ; \\
& J_{4}^{5}=\frac{\pi\left(\delta_{4}^{5}\right)^{4}}{4}=\frac{\pi}{4}(0,44 \delta)^{4}=0,0094 \pi \delta^{4} ; \\
& J_{5}^{5}=\frac{\pi\left(\delta_{5}^{5}\right)^{4}}{4}=\frac{\pi}{4}(0,28 \delta)^{4}=0,0015 \pi \delta^{4} .
\end{aligned}
$$

Przy założeniu $E_{S} J_{1}^{5}=E J$ mamy: $E_{S} J_{2}^{5}=0,466 E J, E_{S} J_{3}^{5}=0,181 E J, E_{s} J_{4}^{5}=0,052 E J$ oraz $E_{s} J_{5}^{5}=0,008 E J$.

$$
\begin{aligned}
& \mu_{1}^{5}=\rho_{s} \cdot A_{1}^{5}=\rho_{s} \cdot \pi \cdot\left(\delta_{1}^{5}\right)^{2}=\rho_{s} \cdot \pi \cdot(0,92 \delta)^{2}=0,8464 \pi \rho_{s} \delta^{2} ; \\
& \mu_{2}^{5}=\rho_{s} \cdot A_{2}^{5}=\rho_{s} \cdot \pi \cdot\left(\delta_{2}^{5}\right)^{2}=\rho_{s} \cdot \pi \cdot(0,76 \delta)^{2}=0,5776 \pi \rho_{s} \delta^{2} ; \\
& \mu_{3}^{5}=\rho_{s} \cdot A_{3}^{5}=\rho_{s} \cdot \pi \cdot\left(\delta_{3}^{5}\right)^{2}=\rho_{s} \cdot \pi \cdot(0,6 \delta)^{2}=0,36 \pi \rho_{s} \delta^{2} ; \\
& \mu_{4}^{5}=\rho_{s} \cdot A_{4}^{5}=\rho_{s} \cdot \pi \cdot\left(\delta_{4}^{5}\right)^{2}=\rho_{s} \cdot \pi \cdot(0,44 \delta)^{2}=0,1936 \pi \rho_{s} \delta^{2} ; \\
& \mu_{5}^{5}=\rho_{s} \cdot A_{5}^{5}=\rho_{s} \cdot \pi \cdot\left(\delta_{5}^{5}\right)^{2}=\rho_{s} \cdot \pi \cdot(0,28 \delta)^{2}=0,0784 \pi \rho_{s} \delta^{2} .
\end{aligned}
$$

Analogicznie przy założeniu $\mu_{1}^{5}=\mu$ otrzymujemy: $\mu_{2}^{5}=0,682 \mu, \mu_{3}^{5}=0,425 \mu, \mu_{4}^{5}=0,229 \mu$ oraz $\mu_{5}^{5}=0,093 \mu$.

Dla poszczególnych cześci słupa wyznaczono parametry: $v_{1}^{5}=1,2 v, v_{2}^{5}=1,3 v, v_{3}^{5}=1,5 v, v_{4}^{5}=1,7 v$ oraz $v_{5}^{5}=2,2 v$. W przypadku podziału słupa na pięć części o stałych przekrojach macierz sztywności przyjmie postać

$$
\mathbf{M}=\left[m_{i j}\right] \text {, dla } i, j=1,2,3,4,5,6,7,8,
$$

gdzie:

$$
\begin{aligned}
& m_{11}=0,833 \alpha(1,2 v)+0,388 \alpha(1,3 v) ; m_{12}=0,388 \beta(1,3 v) ; m_{13}=0 ; m_{14}=0 ; \\
& m_{15}=-0,694 \vartheta(1,2 v)+0,324 \vartheta(1,3 v) ; m_{15}=-0,324 \delta(1,3 v) ; m_{17}=0 ; m_{18}=0 ; \\
& m_{22}=0,388 \alpha(1,3 v)+0,151 \alpha(1,5 v) ; m_{23}=0,151 \beta(1,5 v) ; m_{24}=0 ; m_{25}=0,324 \delta(1,3 v) ; \\
& m_{26}=-0,324 \vartheta(1,3 v)+0,126 \vartheta(1,5 v) ; m_{27}=-0,126 \delta(1,5 v) ; m_{28}=0 ; \\
& m_{33}=0,151 \alpha(1,5 v)+0,043 \alpha(1,7 v) ; m_{34}=0,043 \beta(1,7 v) ; m_{35}=0 ; m_{36}=0,126 \delta(1,5 v) ; \\
& m_{37}=-0,126 \vartheta(1,5 v)+0,036 \vartheta(1,7 v) ; m_{38}=-0,036 \delta(1,7 v) ; \\
& m_{44}=0,043 \alpha(1,7 v)+0,007 \alpha "(2,2 v) ; m_{45}=0 ; m_{46}=0 ; m_{47}=0,036 \delta(1,7 v) ; \\
& m_{48}=-0,036 \vartheta(1,7 v)+0,006 \vartheta "(2,2 v) ; m_{55}=0,579 \gamma(1,2 v)+0,270 \gamma(1,3 v) ; \\
& m_{56}=-0,270 \varepsilon(1,3 v) ; m_{57}=0 ; m_{58}=0 ; m_{66}=0,270 \gamma(1,3 v)+0,105 \gamma(1,5 v) ;
\end{aligned}
$$


$m_{67}=-0,105 \varepsilon(1,5 v) ; m_{68}=0 ; m_{77}=0,105 \gamma(1,5 v)+0,030 \gamma(1,7 v) ; m_{78}=-0,030 \varepsilon(1,7 v)$;

$m_{88}=0,030 \gamma(1,7 v)+0,005 \gamma^{\prime \prime}(2,2 v)$ oraz $m_{i j}=m_{j i}$, dla $i, j=1,2,3,4,5,6,7,8$.

\section{WYNIKI}

W wyniku obliczeń numerycznych otrzymano trzy pierwsze częstości drgań własnych dla każdego z rozpatrywanych wariantów. Wyniki otrzymano za pomocą programu Mathematica.

\section{Wariant 1}

Trzy pierwsze miejsca zerowe wyznacznika przyjmują wartości:

$v_{01}^{1}=0,313$;

$v_{02}^{1}=0,782$;

$v_{03}^{1}=1,309$.

Zatem trzy pierwsze częstości drgań własnych to:

$$
\begin{aligned}
& \omega_{01}^{1}=\left(v_{01}^{1}\right)^{2} \sqrt{\frac{E J}{\mu \delta^{4}}}=0,313 \sqrt{\frac{E_{s} \cdot 0,0324 \pi \delta^{4}}{0,36 \pi \rho_{s} \delta^{2} \cdot \delta^{4}}}=0,029 \sqrt{\frac{E_{s}}{\rho_{s} \delta^{2}}} ; \\
& \omega_{02}^{1}=\left(v_{02}^{1}\right)^{2} \sqrt{\frac{E J}{\mu \delta^{4}}}=0,183 \sqrt{\frac{E_{s}}{\rho_{s} \delta^{2}}} ; \\
& \omega_{03}^{1}=\left(v_{01}^{1}\right)^{2} \sqrt{\frac{E J}{\mu \delta^{4}}}=0,514 \sqrt{\frac{E_{s}}{\rho_{s} \delta^{2}}} .
\end{aligned}
$$

\section{Wariant 2}

Trzy pierwsze miejsca zerowe wyznacznika macierzy sztywności przyjmują wartości:

$v_{01}^{2}=0,380$;

$v_{02}^{2}=0,646$;

$v_{03}^{2}=1,531$.

Zatem trzy pierwsze częstości drgań własnych to:

$$
\begin{aligned}
& \omega_{01}^{2}=\left(v_{01}^{2}\right)^{2} \sqrt{\frac{E J}{\mu \delta^{4}}}=0,380^{2} \sqrt{\frac{E_{s} \cdot 0,1024 \pi \delta^{4}}{0,64 \pi \rho_{s} \delta^{2} \cdot \delta^{4}}}=0,058 \sqrt{\frac{E_{s}}{\rho_{s} \delta^{2}}} ; \\
& \omega_{02}^{2}=\left(v_{02}^{2}\right)^{2} \sqrt{\frac{E J}{\mu \delta^{4}}}=0,167 \sqrt{\frac{E_{s}}{\rho_{s} \delta^{2}}} ; \\
& \omega_{03}^{2}=\left(v_{01}^{2}\right)^{2} \sqrt{\frac{E J}{\mu \delta^{4}}}=0,938 \sqrt{\frac{E_{s}}{\rho_{s} \delta^{2}}} .
\end{aligned}
$$

\section{Wariant 3}

Trzy pierwsze miejsca zerowe wyznacznika macierzy sztywności przyjmują wartości:

$v_{01}^{3}=0,390$;

$v_{02}^{3}=0,652$;

$v_{03}^{3}=1,512$. 
Zatem trzy pierwsze częstości drgań własnych to:

$$
\begin{aligned}
& \omega_{01}^{3}=\left(v_{01}^{3}\right)^{2} \sqrt{\frac{E J}{\mu \delta^{4}}}=0,390^{2} \sqrt{\frac{E_{s} \cdot 0,1410 \pi \delta^{4}}{0,751 \pi \rho_{s} \delta^{2} \cdot \delta^{4}}}=0,066 \sqrt{\frac{E_{s}}{\rho_{s} \delta^{2}}} ; \\
& \omega_{02}^{3}=\left(v_{02}^{3}\right)^{2} \sqrt{\frac{E J}{\mu \delta^{4}}}=0,184 \sqrt{\frac{E_{s}}{\rho_{s} \delta^{2}}} ; \\
& \omega_{03}^{3}=\left(v_{01}^{3}\right)^{2} \sqrt{\frac{E J}{\mu \delta^{4}}}=0,991 \sqrt{\frac{E_{s}}{\rho_{s} \delta^{2}}} .
\end{aligned}
$$

\section{Wariant 4}

Trzy pierwsze miejsca zerowe wyznacznika macierzy sztywności przyjmują wartości:

$v_{01}^{4}=0,407$;

$v_{02}^{4}=0,681$;

$v_{03}^{4}=1,002$.

Zatem trzy pierwsze częstości drgań własnych to:

$$
\begin{aligned}
& \omega_{01}^{4}=\left(v_{01}^{4}\right)^{2} \sqrt{\frac{E J}{\mu \delta^{4}}}=0,407^{2} \sqrt{\frac{E_{s} \cdot 0,1640 \pi \delta^{4}}{0,81 \pi \rho_{s} \delta^{2} \cdot \delta^{4}}}=0,075 \sqrt{\frac{E_{s}}{\rho_{s} \delta^{2}}} ; \\
& \omega_{02}^{4}=\left(v_{02}^{4}\right)^{2} \sqrt{\frac{E J}{\mu \delta^{4}}}=0,209 \sqrt{\frac{E_{s}}{\rho_{s} \delta^{2}}} ; \\
& \omega_{03}^{4}=\left(v_{01}^{4}\right)^{2} \sqrt{\frac{E J}{\mu \delta^{4}}}=0,452 \sqrt{\frac{E_{s}}{\rho_{s} \delta^{2}}} .
\end{aligned}
$$

\section{Wariant 5}

Trzy pierwsze miejsca zerowe wyznacznika macierzy sztywności przyjmują wartości:

$v_{01}^{5}=0,422$;

$v_{02}^{5}=0,695$;

$v_{03}^{5}=1,025$.

Zatem trzy pierwsze częstości drgań własnych to:

$$
\begin{aligned}
& \omega_{01}^{5}=\left(v_{01}^{5}\right)^{2} \sqrt{\frac{E J}{\mu \delta^{4}}}=0,422 \sqrt{\frac{E_{s} \cdot 0,1791 \pi \delta^{4}}{0,8464 \pi \rho_{s} \delta^{2} \cdot \delta^{4}}}=0,082 \sqrt{\frac{E_{s}}{\rho_{s} \delta^{2}}} ; \\
& \omega_{02}^{5}=\left(v_{02}^{5}\right)^{2} \sqrt{\frac{E J}{\mu \delta^{4}}}=0,222 \sqrt{\frac{E_{s}}{\rho_{s} \delta^{2}}} ; \\
& \omega_{03}^{5}=\left(v_{01}^{5}\right)^{2} \sqrt{\frac{E J}{\mu \delta^{4}}}=0,483 \sqrt{\frac{E_{s}}{\rho_{s} \delta^{2}}} .
\end{aligned}
$$




\section{PODSUMOWANIE}

Choć istnieje wiele metod wyznaczania częstości drgań własnych słupów o zmiennym przekroju poprzecznym, w tym również metody ścisłe, to w celach inżynierskich uzasadnione wydaje się stosowanie metod przybliżonych. Otrzymane wartości pierwszych częstości drgań własnych porównano z wartościami otrzymanymi inną metodą przybliżoną, tj. metodą Rayleigh'a (Bagdasaryan, Chalecki, Gierasimiuk, Jaworski i Szlachetka, 2018), wyznaczając błąd względny ze wzoru

$$
\Delta=\left|\frac{\omega_{1}^{R}-\omega_{1}^{M M S}}{\omega_{1}^{R}}\right| \cdot 100
$$

W tabeli podano zestawienie pierwszych częstości drgań własnych wyznaczonych metodą macierzy sztywności, pierwszą częstość drgań własnych rozpatrywanego słupa otrzymaną metodą Rayleigh'a oraz obliczone błędy względne.
$\mathrm{Z}$ wyników przedstawionych $\mathrm{w}$ tabeli wynika, iż rozwiązania przydatne do celów inżynierskich (błąd poniżej 5\%) otrzymuje się przy podziale słupa na przynajmniej pięć przedziałów.

\section{PIŚMIENNICTWO}

Bagdasaryan, V., Chalecki, M., Gierasimiuk, M., Jaworski, J. i Szlachetka, O. (2018). First natural transverse frequency of truncated cone and wedge beams. Acta Sci. Pol. Architectura, 17 (1), 3-12.

Caruntu, D. I. (2009). Dynamic modal characteristics of transverse vibrations of cantilevers of parabolic thickness. Mechanics Research Communications, 36 (3), 391-404.

Naguleswaran, S. (1994). A direct solution for the transverse vibration of Euler-Bernoulli wedge and cone beams. Journal of Sound and Vibration, 172 (3), 289-304.

Szewczyk, M., Kukla, S. i Zamojska, I. (2007). Drgania własne układy płyt kołowych swobodnie podpartych połączonych elementami sprężystymi. Modelowanie Inżynierskie, 33, 153-158.

Tabela. Otrzymane wyniki pierwszej częstości drgań własnych

Table. Obtained results of the first natural frequency

\begin{tabular}{|c|c|c|c|c|c|}
\hline \multirow{2}{*}{$\begin{array}{l}\text { Parametr } \\
\text { Parameter }\end{array}$} & \multicolumn{5}{|c|}{$\begin{array}{l}\text { Podział słupa na } n \text { części } \\
\text { Division of pole per } n \text { pieces }\end{array}$} \\
\hline & $n=1$ & $n=2$ & $n=3$ & $n=4$ & $n=5$ \\
\hline$\omega_{1}^{M M S} \cdot \sqrt{\frac{\rho_{s} \delta^{2}}{E_{s}}}$ & 0,029 & 0,058 & 0,066 & 0,075 & 0,082 \\
\hline$\omega_{1}^{R} \cdot \sqrt{\frac{\rho_{s} \delta^{2}}{E_{s}}}$ & & & 0,086 & & \\
\hline$\Delta[\%]$ & 66,3 & 32,6 & 23,3 & 12,8 & 4,7 \\
\hline
\end{tabular}

\title{
NATURAL FREQUENCY OF A POLE WITH A VARIABLE CROSS-SECTION
}

\begin{abstract}
In the paper natural frequencies of a pole with a variable cross-section were obtained. The pole was made of a homogeneous, elastic material. Solutions were obtained by approximation of the continuous change of the cross-section by changing by steps. The results are compared with the results obtained with Rayleigh's method.
\end{abstract}

Key words: natural frequency, variable cross-section, stiffness matrix 